\title{
Das System der aspektuell-temporalen Formen des modernen Russischen aus der Sicht der Markiertheitstheorie *
}

\author{
Wladimir D. Klimonow \\ Zentrum für Allgemeine Sprachwissenschaft, Sprachtypologie und Universalienforschung \\ Jägerstraße 10/11, D-10117 Berlin \\ E-Mail: klimonov@zas.gwz-berlin.de
}

\section{System der aspektuell-temporalen Formen des russischen Verbs}

Die Kategorie des Tempus existiert in der russischen Sprache der Gegenwart nur in Verbindung mit der Kategorie des Aspekts, was in Aspekt-Tempus-Formen des Verbs zum Ausdruck kommt. Die Kategorie des Tempus interagiert folglich mit der Kategorie des Aspekts. Als Produkt dieses Zusammenwirkens treten im Indikativ die 5 in Schema 1.1. genannten aspektuell-temporalen Formen auf.

Die Frage nach dem Status der Form vom Tyр расскажу bleibt in der linguistischen Literatur strittig. Traditionell wird diese Form als perfektives oder einfaches Futur aufgefaßt, vgl. Pr 1980: 626, RSG 1988: 91. A. V. Isačenko (1976 [1960]: 224-229) betrachtet die Form vom Tyр расскажу als Form des perfektiven Präsens mit der allgemeinen Bedeutung der nichtaktuellen Handlung. Unberücksichtigt bleibt die Tatsache, daß diese Form auch die Bedeutung des aktuellen Präsens aufweist. Die futurale Bedeutung dieser Form versteht A. V. Isačenko als Sonderfall der nichtaktuellen Bedeutung. Die Autoren der tschechischen Akademiegrammatik des Russischen (РГ 1979: 166-167) sehen die Form vom Typ pacскажy als homonym an, d. h. sie sprechen vom Synkretismus der markierten Form des perfektiven Futurs (расскажу ${ }_{2}$ )

\footnotetext{
"Die vorliegende Untersuchung ist im Rahmen des von der Deutschen Forschungsgemeinschaft geförderten Projekts "Markiertheitsrelationen in Tempus-Systemen slavischer Sprachen" entstanden. Sie wurde im Zentrum für Allgemeine Sprachwissenschaft, Sprachtypologie und Universalienforschung (Berlin) am 20. April 1999 präsentiert. Für anregende Diskussion danke ich ganz herzlich allen an der Aussprache beteiligten Kollegen, vor allem Prof. Dr. Michail Kotin, Dr. Brigitta Haftka, Dr. Ivanka Schick und Ljudmila Geist. Mein besonderer Dank gilt den Leitern des Projekts Prof. Dr. Werner Winter und Prof. Dr. Wolfgang Ullrich Wurzel für wertvolle Kommentare und nützliche Hinweise. Für die finanzielle Unterstützung danke ich der Deutschen Forschungsgemeinschaft. Die theoretische Prämissen (Abschnitt 2 dieser Arbeit) wurden in der hier angegebenen Version mit Prof. Dr. Paul Kiparsky (Stanford University) besprochen. Für viele seiner anregenden Gedanken möchte ich mich bei ihm an dieser Stelle ganz herzlich bedanken.
} 
1.1 Aspekt-Tempus-Formen des modernen Russischen

\begin{tabular}{|c|c|c|c|}
\hline Tempora & & \multicolumn{2}{|c|}{ Nicht-Präterita } \\
\hline Aspekte & & Futur & Nicht-Futur \\
\hline Imperfektiv & $\begin{array}{c}\text { Imperfektives Präteritum } \\
\text { рассказывал }\end{array}$ & $\begin{array}{l}\text { Imperfektives Futur } \\
\text { буду рассказывать }\end{array}$ & $\begin{array}{c}\text { Imperfektives Präsens } \\
\text { рассказываю }\end{array}$ \\
\hline Perfektiv & $\begin{array}{l}\text { Perfektives Präteritum } \\
\text { рассказал }\end{array}$ & \multicolumn{2}{|c|}{$\begin{array}{l}\text { Perfektives Nicht-Präteritum } \\
\text { расскажу }\end{array}$} \\
\hline
\end{tabular}

und der unmarkierten Form des perfektiven Präsens (расскажу $)_{1}$ ). M. Guiraud-Weber (1988: 82) betrachtet die Form vom Tyр расскажу als polysem. In der primären Funktion, d. h. im Standardgebrauch, tritt diese Form als Futur auf. In der sekundären Funktion und in der atemporalen Verwendung, d. h. im Nicht-Standardgebrauch,wird diese Form als Präsens aufgefaßt. Die Homonymie oder die Polysemie setzt nach O. P. Rassudova (1982: 82) die Spaltung der Bedeutungen des Präsens und des Futurs voraus. Es gibt aber Kontexte, in denen diese Bedeutungen zusammenfallen, vgl. ОН прекрасно переводит, он и этот специальный текст переведет 'Er übersetzt glänzend, er wird/kann auch diesen Fachtext übersetzen'. Sie schlägt deshalb vor, den Terminus "perfektives Präsens/Futur" zu benutzen. In dieser Untersuchung (Abschnitt 4) wird die Form vom Typ pacскажу als perfektives NichtPräteritum verstanden. Im unmarkierten Gebrauch bezeichnet diese Form das Futur, in der markierten Verwendung treten die präsentischen Bedeutungen dieser Form, nämlich das aktuelle und das nichtaktuelle Präsens auf. Die atemporale Verwendung dieser Form wird als spezifische kontextsensitive (d. h. markierte) Interpretation des Präsens ausgelegt. Nach traditioneller Auffassung existiert eine wesentliche allgemein gültige Beschränkung der temporalen Interpretation des perfektiven Nicht-Präteritums: Diese Form kann angeblich niemals als aktuelles Präsens auftreten (vgl. z. B. Isačenko 1976 [1960]: 226-228). J. L. Bybee und Ö. Dahl (1989: 87) formulieren die Restriktion auf das temporal-referentielle Potential des perfektiven Aspekts als "ausschließlich nichtpräsentische Referenz" (non-present reference only). Es wird gezeigt werden, daß es solche Kontexte gibt, in denen das perfektive Nicht-Präteritum die Bedeutung des aktuellen Präsens aufweist (vgl. Abschn. 3.4.1.2.1). Es existieren zwischen den einzelnen slavischen Sprachen einige Variationen in der temporalen Interpretation der Form des perfektiven Nicht-Präteritums. Diese Form hat z. B. im Bulgarischen nur die Bedeutung der nichtspezifizierten temporalen Referenz.

Im folgenden wird zuerst das Modell vorgestellt, das als theoretische Grundlage für die Betrachtung der Semantik der Aspekt-Tempus-Formen dient (Abschnitt 2). Danach werden die 
syntagmatischen Markiertheitsrelationen der einzelnen aspektuell-temporalen Formen im Rahmen dieses Modells analysiert (Abschnitt 3). Im Abschnitt 4 werden die paradigmatischen Relationen zwischen den Aspekt-Tempus-Formen erörtert. Abschließend (Abschnitt 5) wird das Fazit aus der Untersuchung gezogen.

\section{Theoretische Prämissen}

\section{$2.1 \quad$ Gliederung der aspektuell-temporalen Information}

Als Ausgangskonzept für die Betrachtung der Semantik der aspektuell-temporalen Formen des modernen Russischen dient das dreigliedrige Koordinatensystem, das von H. Reichenbach (1966: 288) stammt: $\mathrm{E}=$ point of the event 'Ereigniszeitpunkt', $\mathrm{S}=$ point of speech 'Sprechzeitpunkt', $\mathbf{R}=$ point of reference 'Referenzzeitpunkt'. Einige Sprachwissenschaftler (darunter auch Paul Kiparsky (1998: 37-39)) führen noch eine vierte Größe $\mathrm{P}=$ perspective time (= Jetzt-Zeitpunkt der temporalen Deixis) ein. Die Funktion von $\mathrm{P}$ wird in der vorgeschlagenen Analyse zwischen $R$ und $S$ verteilt. Die Relationen $E, R$ und $S$ zueinander sind in dem von mir vorgeschlagenen Schema 2.1 als Dreieck dargestellt.

\subsection{Unterteilung der aspektuell-temporalen Information}

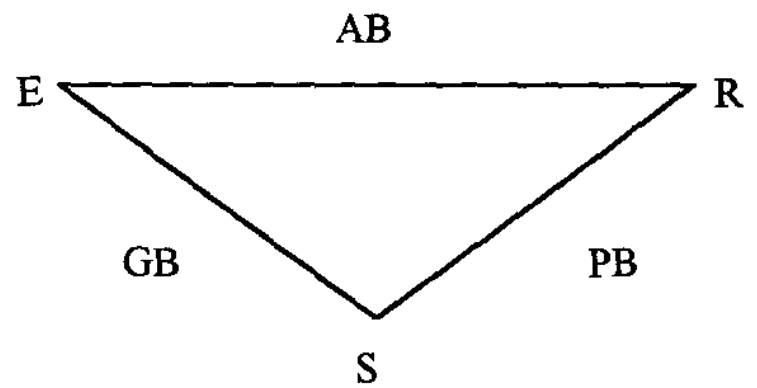

Die aspektuell-temporale Information besteht aus einer aspektuellen Bedeutung $\mathrm{AB}$ (= die Relation $E$ zu R), aus einer systemhaften, d. h. sprachlich determinierten temporal-grammatischen Bedeutung GB (= die Relation $\mathrm{E} z \mathrm{zu}$ ) und einer kontextbedingten, d. h. temporalpragmatischen Bedeutung PB (= die Relation $\mathrm{R}$ zu S).

\section{$2.2 \quad$ Aspektuelle Bedeutung}

Die Relation E zu R definiert die aspektuelle Bedeutung (AB). Der Referenzzeitpunkt $R$ ist die temporale Lokalisierung der Bezugssituation, von der aus das Ereignis $E$ gesehen wird. $R$ ist eine latente Größe, die im Satz mit Hilfe von adverbiellen Bestimmungen spezifiziert werden kann: 
'Um 11 Uhr war Peter (noch) beim Lesen dieses Artikels'

K 12 часам (R) Петр (уже) прочитал (pf. А.) эту статью

'Zu 12 Uhr hatte Peter diesen Artikel (schon) gelesen'

Das Zeitintervall R (в11 часов 'um 11 Uhr') im Beispiel (2-1) mit dem Verb читать 'lesen' im imperfektiven Aspekt (ipf. A.) ist im Zeitintervall E (war beim Lesen), das als Summe der'Intervalle $E_{i}$ aufgefaßt wird $\left(U E_{i}\right)$, enthalten: $R \subset U E_{i}$. Das Lesen des Artikels erstreckt sich auf die Zeit vor $11 \mathrm{Uhr}$, um $11 \mathrm{Uhr}$ und nach $11 \mathrm{Uhr}$. Das Zeitintervall $\mathrm{R}$ wird in diesem Beispiel als gleichzeitig mit einem bestimmten Intervall $E_{i}$ in der Vergangenheit dargestellt: $E_{i}=R$. Im Beispiel (2-2) mit dem Verb прочитать 'lesen' im perfektiven Aspekt (pf. A.) befindet sich das Zeitintervall R (к 12 часам 'zu 12 Uhr') außerhalb des Verbalgeschehens $\mathrm{E}$ (lesen): $\mathrm{R} \not \mathrm{E}$ : Das Lesen des Artikels $(=E)$ ist irgendwann vor $12 \mathrm{Uhr}(\mathrm{R})$ abgeschlossen $(\mathrm{E}<\mathrm{R})$. Die temporalen Bedeutungen der Gleichzeitigkeit $(E=R)$ und der Vorzeitigkeit $(E<R)$ sind von den allgemeinen Bedeutungen des ipf. A. und entsprechend des pf. A. abgeleitet. Die Perfektiva (Pf.) bezeichnen die Ganzheitlichkeit oder die Totalität ([+TOT]) der Handlung, die die Anfangsgrenze und die Endgrenze einschließt:

Петр прочитал этот роман (ог начала доконца)

'Peter hat diesen Roman (vom Anfang bis zum Ende) (durch)gelesen'

Die Imperfektiva (Ipf.) weisen die Bedeutung der Nicht-Ganzheitlichkeit oder der NichtTotalität ([-TOT]) der Handlung auf, die die Anfangsgrenze und die Endgrenze ausschließt:

\section{Петр читал этот роман (где-тов середине)}

'Peter war beim Lesen dieses Romans (irgendwo in der Mitte)

Das perfektive Glied der Aspektopposition mit Einschluß der Handlungsgrenzen wird als markiertes, d. h. komplexeres Glied der aspektuellen Opposition angesehen, während das imperfektive Glied ohne Bezug zu seinen Grenzpunkten als unmarkiertes, d. h. weniger komplexes Glied dieser Opposition betrachtet wird. Die Pf. mit dem Merkmal ([+TOT]) bezeichnen die Abgeschlossenheit der Handlung, d. h. die Vorzeitigkeit der Handlung gegenüber dem Zeitintervall R. Die Ipf. mit dem Merkmal ([-TOT]) drucken die Nicht-Abgeschlossenheit der Handlung und damit die Gleichzeitigkeit der Handlung E mit der Referenzgröße R aus.

\section{$2.3 \quad$ Temporal-grammatische Bedeutung}

Die temporal-grammatische Bedeutung (GB) spezifiziert die Beziehung zwischen $E$ und S. Das Verbalgeschehen $E$ wird zum Sprechzeitpunkt $S$ in einem Jetzt (= Präsens), d. h. bei Gleichzeitigkeit von $E$ und $S(E=S)$ (Beispiel (2-5)), in einem Vorher (= Präteritum), d. h. bei Vorzeitigkeit $E$ vor $S(E<S)$ (Beispiel (2-6)) und in einem Nachher (= Futur), d. h. bei Nachzeitigkeit $\mathrm{E}$ nach $\mathrm{S}(\mathrm{E}>\mathrm{S})$ (Beispiel (2-7)) lokalisiert.

Петр nuшет (Präsens) сейчас (R) это письмо

'Peter schreibt jetzt diesen Brief 


\title{
2.4 Temporal-pragmatische Bedeutung
}

Die temporal-pragmatische Bedeutung (PB), die durch die Relation R zu S gekennzeichnet ist, ermittelt die temporale Interpretation des Satzes in bestimmten Kontexten. In unmarkierten Kontexten, d. h. im Standardgebrauch, fallen GB und PB zusammen, vgl. $\mathrm{E}=\mathrm{S}$ und $\mathrm{R}=\mathrm{S}$ im Beispiel (2-5), $\mathrm{E}<\mathrm{S}$ und $\mathrm{R}<\mathrm{S}$ im Beispiel (2-6) und $\mathrm{E}>\mathrm{S}$ und $\mathrm{R}>\mathrm{S}$ im Beispiel (2-7). Als formale Indikatoren der GB und der PB treten in diesen Beispielen die Verbformen (kursiv) und entsprechend die adverbiellen Bestimmungen (unterstrichen) auf. In markierten Kontexten, d. h. im Nicht-Standardgebrauch, sind GB und $P B$ nicht gleich, vgl. $E=S$ und $R=S$ im Beispiel (2-8) und $\mathrm{E}=\mathrm{S}$ und $\mathrm{R}>\mathrm{S}$ im Beispiel (2-9):

\author{
Мария идет в этот момент (R) в институт \\ 'Maria ist in diesem Augenblick auf dem Wege ins Institut' \\ Мария идет через час (R) в институт \\ 'Maria wird in einer Stunde ins Institut gehen'
}

Die Relation E zu S, die durch die Verbform идет 'ist auf dem Wege' ausgedrückt ist, bleibt in beiden Beispielen ((2-8) und (2-9)) gleich: $\mathrm{E}=\mathrm{S}$. Die Relation $\mathrm{R}$ zu $\mathrm{S}$ ist aber unterschiedlich. Die Referenzzeit $R$ в этот момент 'in diesem Augenblick' im Beispiel (2-8) ist in der Gegenwart $(R=S)$ lokalisiert, während $R$ через час 'in einer Stunde' im Beispiel (2-9) in die Zukunft verlagert ist $(R>S)$. Die Verschiebung der PB gegenüber der GB hat die Reinterpretation der GB der Äußenung zur Folge: Die GB (= die Bedeutung des Präsens im Beispiel (2-9)) wird ausgeblendet und durch die PB (= die Bedeutung des Futurs) ersetzt. Die AB und die GB sind immer kontextunabhängig, d. h. konstant, während die PB kontextsensitiv, d. h. variabel ist.

\section{Markiertheitsrelationen der aspektuell-temporalen Formen auf der syntagmati- schen Ebene}

\subsection{Das imperfektive Präteritum vom Typ рассказывал 'ich erzählte'}

\subsubsection{Temporale Bedeutungen}

\subsubsection{Unmarkierte temporale Bedeutung}

Das imperfektive Präteritum (ipf. Prät.) vom Tур рассказьвал 'ich erzählte' bezeichnet in temporaler Hinsicht im unmarkierten, d. h. natürlichen, typischen, standardisierten Gebrauch ein vergangenes Geschehen, das von der Gegenwart, d. h. vom Sprechzeitpunkt S, vollkommen losgelöst ist. Die temporal-grammatische Bedeutung (GB) dieser Form wird als $\mathrm{E}<\mathrm{S}$ und 
die temporal-pragmatische Bedeutung $(\mathrm{PB})$ als $\mathrm{R}<\mathrm{S}$ repräsentiert. Die zeitliche Getrenntheit ([+GETR(ENNTHEIT)]) des Referenzzeitpunktes $\mathrm{R}$ von $\mathrm{S}(\mathrm{R} \not \mathrm{S})$ wird als allgemeine temporale Bedeutung des ipf. Prät. betrachtet.
a. Мария нравилась (ipf. A.) Петру 'Maria gefiel Peter'
b. Мария тегда нравилась (ipf. А.) Петру, но сейчас уже ему не нравитсs
'Maria gefiel damals Peter, aber sie gefallt ihm jetzt nicht mehr'
a. Мария понравилась (pf. А.) Петру
'Maria hat Peter gefallen'
b. Мария тогда понравилась (pf. А.) Петру и нравитсл ему также сейчас
'Maria hat damals Peter gefallen, und sie gefält ihm auch jetzt'
a. Вы подписывались (ipf. А.) когда-нибудь на этот журнал? 'Hatten Sie diese Zeitschrift irgendwann einmal abonniert?'
b. Да, я кегда-то подписывался (ipf. А.) на него 'Ja, ich hatte sie vor Zeiten abonniert'
c. Да, я когда-то подписывался (ipf. А.) на него, но сейчас уже не . подписываюсь

'Ja, ich hatte sie vor Zeiten abonniert, aber jetzt aboniere ich sie nicht mehr'
a. Вы уже подписались (pf. А.) на этот журнал? 'Haben Sie diese Zeitschrift schon abonniert?'
b. Да, я на него подписался (pf. A.) 'Ja, ich habe sie abonniert'
c. Да, я на него подписался (pf. А.) недавно и сейчас уже получаю ero
'Ja, ich habe sie vor kurzem abonniert und beziehe sie jetzt schon'

\begin{abstract}
Das perfektive Präteritum (pf. Prät.) vom Тур понравилась 'hat gefallen' im Beispiel (3-1-2) a. hat die perfektische Bedeutung, d. h. es wird als (3-1-2) b. mit dem Merkmal [-GETR] aufgefaßt. Das ipf. Prät. vom Тур нравилась 'gefiel' im Beispiel (3-1-1) a. hat dagegen die aoristische (= konkret-faktische) Bedeutung, d. h. es wird im Kontrast zu (3-1-2) a. als (3-1-1) b. mit dem Merkmal [+GETR] ausgelegt. Im Beispiel (3-1-3) drückt das ipf. Prät. eine ferner in der Vergangenheit liegende Handlung aus. Die Bedeutung der vorvergangenen Zeit wird im Deutschen durch das Plusquamperfekt wiedergegeben. Das pf. Prät. im Beispiel (3-1-4) weist dagegen perfektische Bedeutung auf, die im Deutschen durch die Form des Perfekts manifestiert wird.
\end{abstract}

\title{
3.1.1.2 Markierte temporale Bedeutungen
}

In der markierten, d. h. nichtnatürlichen, nichttypischen, nichtstandardisierten Verwendung drückt das ipf. Prät. die präsentische oder die futurische Bedeutung aus. 


\subsection{Präsens}

Die präsentische Bedeutung des ipf. Prät. tritt in der saloppen Umgangssprache beim expressiv-emotionalen Ausdruck des Mißachtens seitens des Sprechers in Erscheinung:

$$
\begin{aligned}
& \text { Плевал (Präteritum) я на эти сплетни != Я плюю (Präsens) на эти } \\
& \text { сплетни } \\
& \text { 'Ich pfeife auf dieses Gerede' }
\end{aligned}
$$

Die Negierung der Handlung in der Gegenwart wird durch ihre ironische Kontrastierung in der Vergangenheit mit Hilfe des ipf. Prät wiedergegeben:

Боялся (Präteritum) я его, как же ! = Я его не боюсь (Präsens).

'Als ob ich vor ihm Angst hätte!' / 'Ich habe keine Angst vor ihm'

Die präteritale grammatische Bedeutung $(\mathrm{GB})$ des ipf. Prät. $(\mathrm{E}<\mathrm{S})$ stimmt nicht mit der präsentischen pragmatischen Deutung dieser Form $(R=S)$ überein. Die GB wird bei solcher Kontroverse ausgeblendet. Die PB bestimmt dann die temporale Interpretation einer Äußerung.

\subsection{Futur}

Die Bedeutung des Futurs tritt in der Kette von präteritalen Handlungen dann auf, wenn eine darauf folgende Handlung als die nächste Zukunft in bezug auf eine frühere Handlung aufgefaßt wird. Eine bevorstehende Handlung hat dann eine nezessitative modale Bedeutung, vgl.

Студент много работал, потому что он сдавал ( = должен был сдавать) экзамены

Der Student arbeitete viel, weil er Prüfungen machte (= machen mußte) Петр консультировался у своего профессора, который на следуюший день уезжал (= должен был уехать) в отпуск 'Peter hat seinen Professor, der am nächsten Tag in Urlaub fuhr (= fahren mußte) um Rat gefragt'

Die GB E < S, die im Widerspruch zur PB R > S steht, wird getilgt. In diesem Kontext bleibt als temporale Bedeutung nur die Äußerungsbedeutung $\mathrm{R}>\mathrm{S}$.

\subsubsection{Aspektuelle Bedeutungen}

\subsubsection{Unmarkierte aspektuelle Bedeutung}

Als unmarkierte aspektuelle Bedeutung des ipf. Prät. wird die Bedeutung der Nicht-Totalität der Handlung ([-TOT]) angenommen. Diese Bedeutung wird primär als konkret-prozessuale 
(oder durative) Bedeutung manifestiert. Das ipf. Prät. drückt dann eine konkrete Eizelhandlung in ihrem Verlauf aus, die in der Vergangenheit lokalisiert ist:

Мария читала эту газету, когдапришел Петр

'Maria las diese Zeitung, als Peter kam'

Dementsprechend wird die aspektuelle Bedeutung (AB) dieser Form auf der Zeitachse E - R S (vgl. Schema (2-1) als Relation der Gleichzeitigkeit des Referenzzeitpunkts R und des Ereigniszeitpunkts $\mathrm{E}(\mathrm{R}=\mathrm{E})$ definiert (vgl. Beispiel (2-2)).

\title{
3.1.2.2 Markierte aspektuelle Bedeutungen
}

Die markierten aspektuellen Bedeutungen des ipf. Prät. treten in speziellen Kontexten, d. h. unter besonderen, einschränkenden Bedingungen des Gebrauchs dieser Form auf. Als markierte aspektuelle Bedeutungen werden die Bedeutung der Multiplizierung der Handlung, die Bedeutung der Totalität der Handlung ([+TOT]) und die Bedeutung der Nicht-Spezifizierung des Merkmals der Totalität der Handlung ([ \pm TOT]) aufgefaßt.

Die Form des ipf. Prät. kann die folgenden kontextsensitiven Handlungen bezeichnen: die iterativen, nämlich die unbegrenzt-iterativen (Beispiel (3-1-10)) und die begrenzt-iterativen (Beispiel (3-1-11)), die usuellen (oder die habituellen) (Beispiel (3-1-12)), die permanenten (oder die qualifizierenden) (Beispiel (3-1-13)), die possibilitativen (oder die potenziellen) (Beispiel (3-1-14)).

\author{
Мария время от времени перечитывала своих любимых поэтов \\ 'Maria las ab und zu ihre Lieblingsdichter' \\ Мария дважды читала этот рассказ \\ 'Maria hat diese Erzählung zweimal gelesen' \\ Мария по утрам читала эту газету \\ 'Maria las (immer) morgens diese Zeitung' \\ Мария всегда одевалась по последней моде \\ 'Maria kleidete sich immer nach der neuesten Mode' \\ Мария танцевала аргентинское танго \\ 'Maria tanzte argentinischen Tango'
}

Diese Handlungen haben einen gemeinsamen Nenner: Sie drücken mehrmalige (oder multiplikative) Ereignisse aus. Die Bezeichnung der multiplikativen Handlungen macht die sekundäre, d. h. spezielle aspektuelle Bedeutung des ipf. Prät. aus.

Es gibt Kontexte, in denen die Form des ipf. Prät. die Bedeutung der Totalität der Handlung ([+TOT]) ausdrücken kann, d. h. anstelle der Form des pf. Prät. gebraucht wird. 
Профессор только что вернулся из университета, он читал (= прочитал) там очередную лекцию

'Der Professor ist gerade von der Universität zurückgekommen, er hat dort die planmäßige Vorlesung gehalten'
a. Кто строил (= построил) это высотное здание?

'Wer hat dieses Hochhaus gebaut?'

b. $\quad$ Это высотное здание строили (= построили) наши строители

'Dieses Hochhaus haben unsere Bauleute gebaut'

Im Beispiel (3-1-15) wird eher die Form des pf. Prät. erwartet. Der ganze Kontext und insbesondere die Adverbialbestimmung уже 'schon' bezeichnen die Vollendung der Handlung (= das Gegessen-Haben). Ähnlich ist die Sachlage im Beispiel (3-1-16): Die Vorlesung hat schon stattgefunden. Das Hochhaus im Beispiel (3-1-17) ist schon fertiggestellt. Das folgt aus dem Kontext. Fokussiert werden die Handlung selbst und ihr Agens. Als markierte aspektuelle Bedeutung (AB) des ipf. Prät. auf der Zeitachse E - R -S (s. Schema (2-1)) tritt die Bedeutung der Vorzeitigkeit des Ereigniszeitpunkts $E$ dem Referenzzeitpunkt $R$ gegenüber $(E<R)$ auf, vgl. Beispiel (2-3).

Die Neutralisierung der aspektuellen Differenzierung, d. h. die Nicht-Spezifizierung des Merkmals der Totalität der Handlung ([† TOT]), wird im Kontext der bloßen Konstatierung einer vergangenen Handlung festgestellt (= die sogenannte abstrakt-faktische oder allgemeinfaktische Bedeutung des ipf. Prät.). Die entsprechende Handlung wird nur genannt. Die aspektuelle Information wird als unwesentlich angesehen und unspezifiziert gelassen.

(3-1-18) a. Петр принимал это лекарство

'Peter hat dieses Arzneimittel eingenommen'

b. Петр принимал (= принял) это лекарство сегодня первый раз

'Peter hat dieses Arzneimittel heute zum ersten Mal eingenommen'

c. Петр принимал (* принял) это лекарство много раз

'Peter hat dieses Arzneimittel mehrmals eingenommen'

(3-1-19) a. Мария читала этот роман

'Maria hat diesen Roman gelesen'

b. Мария когда-то читала этот роман

'Maria hat diesen Roman irgendwann gelesen'

c. Мария много раз читала этот роман

'Maria hat diesen Roman oft / viele Male gelesen'

d. Мария с увлечением читала (= прочитала) этот роман от начала

до конша

'Maria hat diesen Roman mit Begeisterung vom Anfang bis zum Ende (durch)gelesen'

e. Мария читала (*прочитала) этот роман, но не дочитала его до конща

'Maria hat an diesem Roman gelesen, aber sie hat ihn nicht zu Ende gelesen. 
Der Satz (3-1-18) a. ist mehrdeutig. Er kann eine Einzelhandlung (im Sinne des Beispiels (3-118) b.) bezeichnen. Diese Lesart ist mit der Bedeutung des pf. Prät. referenziell identisch. Das Beispiel (3-1-18) a kann man aber auch iterativ (im Sinne der Lesung (3-1-18) c) auffassen. Diese imperfektivische Lesart ist mit der Bedeutung des pf. Prät. inkompatibel. Die Äußerung (3-1-19) a. ist auch nicht eindeutig. Gemeint ist entweder eine Einzelhandlung (Beispiel (3-119) b.) oder eine wiederholte Handlung (Beispiel (3-1-19) c). Eine Einzelhandlung kann ihrerseits als perfektiv (Beispiel (3-1-19) d) oder als imperfektiv (Beispiel (3-1-19) e) interpretiert werden.

\subsection{Das perfektive Präteritum vom Typ paccказал 'ich habe erzählt'}

\subsubsection{Temporale Bedeutungen}

\subsubsection{Unmarkierte temporale Bedeutungen}

Als unmarkierte temporale Bedeutungen des pf. Prät vom Тур рассказал 'ich habe erzählt' treten die Bedeutungen des Perfekts, des Aorists und des Plusquamperfekts auf.

Die perfektische Bedeutung bezeichnet eine in der Vergangenheit abgeschlossenen Handlung, die mit ihrer Auswirkung in die Gegenwart hineinreicht.

$$
\begin{aligned}
& \text { Петр открыл сегодня утром окно, сейчас оно открыто, и в } \\
& \text { комнате стало прохладно } \\
& \text { 'Peter hat heute früh das Fenster geöffnet, jetzt ist es auf, und es ist kühl } \\
& \text { im Zimmer geworden' }
\end{aligned}
$$

Die temporal-grammatische Bedeutung (GB) wird als $\mathrm{E}<\mathrm{S}$ und die temporal-pragmatische Bedeutung (PB) als $\mathrm{R}<\mathrm{S}$ dargestellt. Der Referenzzeitpunkt $\mathrm{R}$ enthält den Sprechzeitpunkt S: R $\supset$ S. Diese Bedeutung weist folglich das Merkmal [-GETR] auf. Die perfektische Bedeutung des pf. Prät. wird traditionell als Hauptbedeutung dieser Form betrachtet (vgl. Виноградов 1972: 443; Домбровский 1971: 92-93; ГСРЛЯ 1970: 358).

Die aoristische (= konkret-faktische) Bedeutung bezeichnet eine konkrete abgeschlossene, in der Vergangenheit lokalisierte Einzelhandlung, die keine Auswirkungen in der Gegenwart hat:

$$
\begin{aligned}
& \text { Вчера Мария встретила свою подругу в театре } \\
& \text { 'Gestern hat Maria ihre Freundin im Theater getroffen' }
\end{aligned}
$$

Die GB und die PB bleiben die gleichen wie bei der perfektiven Bedeutung dieser Form, es ändert sich nur die Wertung des Merkmals der Getrenntheit: Die aoristische Bedeutung ist durch die positive Spezifizierung dieses Merkmals ([+GETR]) gekennzeichnet. In diesem Fall liegt der Sprechzeitpunkt $\mathrm{S}$ außerhalb des Referenzzeitpunktes $\mathrm{R}: \mathrm{R} \not \supset \mathrm{S}$. A. V. Isačenko (1962: 293) betrachtet die Aoristbedeutung als Hauptbedeutung des pf. Prät. 
Die Bedeutung des Plusquamperfekts tritt bei der Verwendung des pf. Prät. dann auf, wenn eine von zwei Handlungen in der Vergangenheit früher als andere stattgefunden hat:

Петр дал сегодня Марии книгу, которую он вчера купил в книжном магазине

'Peter hat Maria heute das Buch gegeben, das er gestern in einer Buchhandlung gekauft hatte'

Die Bedeutung des Plusquamperfekts wird als Variante der aoristischen Bedeutung interpretiert.

\subsubsection{Markierte temporale Bedeutungen}

\subsubsection{1 $\quad$ Futur}

Die Form des pf. Prät. drückt zuweilen vorausgreifend eine Handlung aus, die erst eintreten wird oder soll (praeteritum pro futuro). Im Kontext der expliziten und impliziten Konditionalsätze kann die Form des pf. Prät. die Handlung mit dem vorweggenommenen Resultat in der Zukunft (das sogenannte praeteritum propheticum) bezeichnen:

Если у него козырная карта, то он выиграл 'Wenn er einen Trumpf hat, dann hat er gewonnen (= wird er gewinnen)' Без нашей помоши она пропала 'Ohne unsere Hilfe ist sie verloren (= wird sie verloren sein)'

Das pf. Prät. bezeichnet mit den Bewegungsverben in der Umgangssprache die unmittelbar folgende Zukunft (Beispiel (3-2-6) a)). Die Strukturen dieser Art weisen bestimmte syntaktische Restriktionen auf: Sie erlauben keine Negation (Beispiel (3-2-6) b) und keine adverbiellen Bestimmungen als Erweiterungen (Beispiel (3-2-6) c).
a. Ну, я пошел ! = Я сейчас пойду
Nun gehe ich gleich (wörtlich: ich bin gegangen)'
b. *Hy, я не nowes
* 'Nun bin ich nicht gegangen'
c. * * $\quad *$ бу быстро пошел
* 'Nun bin ich schnell gegangen'

Die ironische Negierung der zukünftigen Handlung wird durch das pf. Prät. im Kontext mit den Partikeln как же, то и оder mit Hilfe der Inversion mit besonderer Intonation ausgedrückt:

Как же, пошла я за него замуж!

'Ich werde ihn niemals heiraten !' 
Die GB E $<$ S wird ausgeblendet. Als temporale Äußerungsbedeutung gilt nur die PB R $>$ S.

\subsection{Atemporale Bedeutung}

Das pf. Prät. wird auch atemporal verwendet. Der Referenzzeitpunkt $R$ bleibt in diesem Fall unspezifiziert: Er kann die Gegenwart, die Vergangenheit und die Zukunft gleichermaßen betreffen. Diese Funktion des pf. Prät kommt in Aphorismen vor:

$$
\begin{aligned}
& \text { Что с возу упало, то пропало } \\
& \text { 'Was von der Fuhre gefallen ist, ging verloren (= ist weg)' }
\end{aligned}
$$

Die gleiche atemporale Bedeutung findet man in der sogenannten multiplikativ-paarigen Konstruktion, die die Wiederholbarkeit des Zyklus aus zwei Ereignissen darstellt (ein Ereignis geht dabei dem anderen voran):

$$
\begin{aligned}
& \text { Каштаны цветут весной, когда только что распустился молодой } \\
& \text { лист } \\
& \text { 'Kastanien blühen im Frühjahr, wenn das junge Laub gerade erst aufge- } \\
& \text { brochen ist' }
\end{aligned}
$$

\subsubsection{Aspektuelle Bedeutungen}

\subsubsection{Unmarkierte aspektuelle Bedeutung}

Als unmarkierte aspektuelle Bedeutung des pf. Prät. tritt die Bedeutung der Ganzheitlichkeit oder der Totalität der Handlung ([+TOT]) auf. Diese Bedeutung wird primär als konkretfaktische Bedeutung manifestiert, d. h. als Bezeichnung einer konkreten in der Vergangenheit abgeschlossenen Einzelhandlung. Die Handlung wird als vollendete Tatsache (in facto) dargestellt (vgl. Beispiel (2-4)). Die aspektuelle Bedeutung (AB) dieser Form auf der Zeitachse $\mathrm{E}-\mathrm{R}-\mathrm{S}$ wird als $\mathrm{E}<\mathrm{R}$ repräsentiert.

\subsubsection{Markierte aspektuelle Bedeutung}

Mit den adverbiellen Bestimmungen der begrenzten Wiederholung vom Тур дважды 'zweimal', пять раз 'fünfmal', несколько раз 'mehrmals' bezeichnet das pf. Prät eine Reihe kontinuierlich aufeinanderfolgender Handlungen, die als Gesamtheit (= die sogenannte summarische Bedeutung) aufgefaßt werden:

Петр несколько раз перечитал это стихотворение, чтобы его запомнить

'Peter hat dieses Gedicht mehrmals gelesen, um es sich einzuprägen' 
Die parallele Form des ipf. Prät. schildert eher sporadische Ereignisse, die voneinander durch größere Intervalle getrennt werden können und die keine summarische Lesart erlauben:

Петр несколько раз перечитывал это стихотворение, потому что оно ему понравилось

'Peter hat dieses Gedicht mehrmals gelesen, weil es ihm gefallen hat'

Die adverbiellen Bestimmungen vom Тур иногда 'manchmal', время от времени 'von Zeit zu Zeit' die speziell auf sporadische Ereignisse referieren, erlauben nicht die Verwendung des pf. Prät.

Петр время от времени перечитывал это стихотворение, потому что оно ему нравилось

'Peter hat dieses Gedicht von Zeit zu Zeit gelesen,weil es ihm gefiel' *Петр время от времени перечитал это стихотворение, потому что оно ему нравилось

'Peter hat dieses Gedicht von Zeit zu Zeit gelesen, weil es ihm gefiel'

\subsection{Das imperfektive Futur vom Typ буду pacсказывать 'ich werde erzählen'}

\subsubsection{Temporale Bedeutungen}

\subsubsection{Unmarkierte temporale Bedeutung}

Das imperfektive Futur (ipf. Fut.) vom Тур буду рассказывать 'ich werde erzählen' bezeichnet normalerweise eine Handlung, die in der Zukunft lokalisiert ist. Die GB wird als E > S und PB als $R>S$ repräsentiert. Das ipf. Fut. weist auf die vollkommene Losgelöstheit des Sprechzeitpunkts S vom Referenzzeitpunkt R ([+GETR]) oder $R \not \supset S$ ) hin. Damit unterscheidet sich das ipf. Fut. vom Тур будет одеваться (vgl. Beispiele unter (3-3-1)) vom perfektiven Futur vom Тур оденется (vgl. Beispiele unter (3-3-2)), das dieses Merkmal unausgedrückt läßt.
a. Мария будет одеваться на банкет
'Maria wird sich zum Festessen ankleiden'
b. Мария будет после работы одеватьсл на банкет
'Maria wird sich nach Dienstschluß zum Festessen ankleiden'
c. *Мария сейчас одевается и скоре уже будет одеваться на банкет *'Maria kleidet sich jetzt gerade an und wird sich gleich zum Festessen ankleiden'
(3-3-2) a. Мария оденется на банкет
'Maria wird sich zum Festessen ankleiden'
'Maria wird zum Festessen angekleidet sein'
b. Мария оденется после работы на банкет
'Maria wird sich nach Dienstschluß zum Festessen ankleiden' 
c. Мария сейчас одевается и скоро уже оденется на банкет 'Maria kleidet sich jetzt gerade zum Festessen an und wird gleich damit fertig sein'

Der Referenzzeitpunkt $R$ der Handlung E im Satz (3-3-1) a wird als getrennt vom Sprechzeitpunkt $S$ ([+GETR]) verstanden (Maria hat in diesem Augenblick (S) noch nicht begonnen, sich anzuziehen), so wie es im Beispiel (3-3-1) b explizit angegeben ist. Im Kontext der NichtGetrenntheit des Referenzzeitpunkts R einer Handlung E vom Sprechzeitpunkt S ([-GETR]) (so wie im Beispiel (3-3-1) c) kann diese Form nicht verwendet werden. Der Referenzzeitpunkt $\mathrm{R}$ der Handlung $\mathrm{E}$ im Satz (3-3-2) a ist in bezug auf den Sprechzeitpunkt $\mathrm{S}$ dagegen nicht festgelegt: In Abhängigkeit vom Kontext kann $R$ als getrennt von $S$ ([+GETR]) (vgl. Beispiel (33-2) b) oder als nichtgetrennt von S ([-GETR]) (vgl. Beispiel (3-3-2) c) gedeutet werden. Im Beispiel (3-3-2) c fallt der Anfang der Handlung mit dem Sprechzeitpunkt $S$ zusammen, und der Abschluß der Handlung wird in die Zukunft verlagert.

\subsubsection{Markierte temporale Bedeutungen}

\subsection{Präsens}

Bei der Annahme über unbekannte Tatsachen werden noch nicht erkennbare Sachverhalte auf die Gegenwart bezogen:

Ему лет хридцать будет

'Er wird dreißig Jahre alt sein'

Die negative Reaktion des Sprechers im Beispiel (3-3-4) vollzieht sich in der Gegenwart:

Еще секретарша будет мне указывать! (= указывает)

'Vielleicht gibt mir noch eine Sekretärin Anweisungen!'

Die GB wird als $E>S$ und die $P B$ als $R=S$ repräsentiert. Als temporale Satzbedeutung gilt die PB R $=$ S.

\subsection{Atemporale Bedeutung}

Das ipf. Fut. wird selten atemporal verwendet:

Пятью пять будет двадцать пять

'Fünf mal fünf macht / ergibt / ist fünfundzwanzig'

In diesem Satz wird die zeitlich nicht lokalisierte allgemeingültige Handlung bezeichnet. Der Referenzzeitpunkt R wird auf der Zeitachse E - R - S nicht festgelegt. 


\subsubsection{Aspektuelle Bedeutungen}

\subsubsection{Unmarkierte aspektuelle Bedeutung}

Die primäre aspektuelle Bedeutung des ipf. Futurs ist die konkret-faktische Bedeutung, d. h. die Bezeichnung einer konkreten Einzelhandlung:

Петр будет завтра сдавать экзамен

'Peter wird morgen die Prüfung ablegen'

Der Referenzzeitpunkt $R$ fällt auf der Zeitachse E - R - S mit dem Ereigniszeitpunkt E zusammen: $\mathrm{R}=\mathrm{E}$.

\subsubsection{Markierte aspektuelle Bedeutungen}

Als sekundäre kontextsensitive Bedeutungen der Form des ipf. Fut. treten die Bedeutungen der multiplikativen Handlungen und die modalen Bedeutungen auf.

Die temporalen Adverbialien vom Тур часто 'oft', редко 'selten', постоянно 'ständig', всегда 'immer' weisen auf iterative bzw. habituelle Handlungen hin:

Теперь якаждый день буду читать (*прочту) эту газету

'Nun werde ich diese Zeitung jeden Tag lesen'

Mit diesen lexikalischen Indikatoren wird nur der ipf. A. gebraucht.

Die Marker der quantitativen Begrenzung einer Handlung vom Тур дважды 'zweimal', несколько раз 'mehrmals' erlauben die Verwendung von beiden Aspekten:

Я буду писать / напишу сегодня нескольке писем

'Ich werde heute mehrere Briefe schreiben'

Die aspektuellen Bedeutungen werden oft von modalen Bedeutungen überlagert. Das ipf. Fut. weist dann die modalen Bedeutungen der Absicht (Beispiel (3-3-9)), des Wunsches (Beispiel (3-3-10)), der Willensäußerung (vgl. die Verweigerung einer Handlung im Beispiel (3-3-11)) auf. Im Kontrast zum perfektiven Futur kann das ipf. Fut. die Bedeutung der Ungewißheit in der Realisierung einer Handlung ausdrücken (Beispiel (3-3-12)).

Вы будете (= намерены) выходить?

'Werden Sie aussteigen? / Haben Sie die Absicht, auszusteigen?'

Что Вы будете (= желаете) заказывать к обеду?

'Was werden (= möchten) Sie zum Mitagessen bestellen?

He бydy (= не xочy) ecmb!

'Ich werde (= will) nicht essen!' 
Вы напишете статью к началу семестра?

'Werden Sie den Artikel zum Anfang des Semesters (fertig)schreiben?'

Буду писать (= буду пытаться), надеюсь, что напишу

'Ich werde schreiben (= versuche es), ich hoffe, daß ich ihn fertigschreibe'

\subsection{Das perfektive Nicht-Präteritum vom Typ pacckaæcy 'ich werde erzählen, ich erzähle'}

\subsubsection{Temporale Bedeutungen}

\subsubsection{Unmarkierte temporale Bedeutung}

Das perfektive Nicht-Präteritum ( pf. Nicht-Prät.) vom Typ расскажу 'ich werde erzählen, ich erzähle' wird kontextunabhängig als perfektives Futur interpretiert.

Петр прочтет эту статью завтра

'Peter wird diesen Artikel morgen durchlesen'

Die GB wird als E $>$ S und PB als R > S repräsentiert. In bezug auf das Merkmal [ $\alpha$ GETR] bleibt diese Form unspezifiziert, vgl. Abschnitt 3.3.1.1 Beispiel (3-3-2).

\subsubsection{Markierte temporale Bedeutungen}

Als markierte temporale Bedeutungen werden die Bedeutungen des aktuellen und des nichtaktuellen Präsens, des Präteritums (= praesens historicum) und die atemporale Bedeutung registriert.

\subsection{Präsens}

Das pf. Nicht-Prät. von performativen Verben weist die Bedeutung des aktuellen Präsens ([+ACT]) im Kontext hic et nunc 'hier und jetzt' auf, vgl. die Beispiele (3-4-2) und (3-4-3). Die Form des pf. Nicht-Prät. unterscheidet sich von der Form des imperfektiven Präsens durch eine voluntative oder desiderative modale ([+MOD]) Bedeutung, die die Absicht des Sprechers kennzeichnet, vgl. die Verwendung dieser Form im Kontext mit modalen Partikeln des Typs пожалуй что 'wohl doch', все-таки 'dennoch' im Beispiel (3-4-4), die auf die Bevorzugung bei der Wahl seitens des Sprechers hinweisen.

Я сразу же Вас на этом месте перебью

'Ich unterbreche Sie gleich jetzt und an dieser Stelle'

На этом я закончу в данный момент свой доклад

'Hiermit beende ich meinen Vortrag'

(Обращение посетителя ресторана к официанту:)

Я закажу / *заказываю пожалуй что / все-таки бифштекс 
(Restaurantbesucher zum Kellner:)

'Ich bestelle wohl doch / dennoch ein Hacksteak'

Die Negierung des zu erwartenden Ergebnisses einer Handlung erstreckt sich auch auf die Gegenwart: Es gibt keine Möglichkeit eine Handlung in diesem Augenblick zu realisieren.

$\begin{array}{ll}\text { (3-4-5) } & \text { Никак не найду своей тетради } \\ & \text { 'Ich finde mein Неft nicht = Ich kann mein Heft (absolut) nicht finden' } \\ \text { (3-4-6) } & \begin{array}{l}\text { Не придумаю, как выйти из этого положения } \\ \text { 'Ich finde keinen Ausweg aus dieser Lage }\end{array} \\ \text { (3-4-7) } & \begin{array}{l}\text { Не разберешь, сколько ему лет } \\ \text { 'Man wеiß nicht, wie alt er ist }\end{array} \\ \text { (3-4-8) } & \begin{array}{l}\text { Вас и не узнаешь } \\ \text { 'Man kann Sie nicht wiedererkennen' }\end{array}\end{array}$

Die GB wird als $\mathrm{E}>\mathrm{S}$ und die $\mathrm{PB}$ als $\mathrm{R}=\mathrm{S}$ repräsentiert. Die $\mathrm{GB}$ wird dabei ausgeblendet.

Das pf. Nicht-Prät. kann auch als nichtaktuelles Präsens ([-ACT]) gebraucht werden. Das usuelle (oder habituelle) Präsens und das permanente (oder qualifizierende) Präsens illustrieren die nichtaktuelle Verwendung dieser Form:

(3-4-9) Иногда я так устаю, что приду домой, поужинаю и сразу засну 'Manchmal bin ich so müde, daß ich nach Hause komme, Abendbrot esse und sofort einschlafe'

(3-4-10) Петр человек решительный. Он всегда ответит напрямик 'Peter ist ein resoluter Mensch. Er antwortet immer direkt'

\subsection{Präteritum (= praesens historicum)}

Das historische Präsens wird angewendet, um eine objektiv vergangene Handlung in die Gegenwart zu übertragen und sie dadurch wirkungsvoll und lebendig darzustellen.

Днем она большею частью дремала. Cядет в кресло перед столом (...) и дремлет (М. Салтыков-Щедрин).

'Am Tage schlummerte sie meistens. Sie setzt sich in den Sessel vor dem Tisch (...) und schlummert.'

Im Beispiel (3-4-11) werden die Formen des ipf. Nicht-Prät. und des ipf. Präsens in der Funktion des historischen Präsens verwendet.

In Verbindung mit den Partikeln (да) как, как вдруг bezeichnet das ipf. Nicht-Prät. eine plötzliche überraschende Handlung: 

захлебывась. Герасим гдядел, глядел да как засмеется вдруг (...) (И. С. Тургенев)

'Das Hündchen fing plötzlich gierig zu trinken an, schnaufte, zitterte und verschluckte sich dabei. Gerasim schaute und schaute und lachte plötzlich los'

Mit der Partikel бывало steht das pf. Nicht-Prät. zur Bezeichnung gewohnheitsmäßig in ferner Vergangenheit vollzogener Handlungen:

$$
\begin{aligned}
& \text { Что за девка то была! Бывало, кто ни проедет, всякий похвалит, } \\
& \text { никто не осудит. (А. С. Пушкин) } \\
& \text { 'Was war das für ein Mädchen! Wer auch immer hier des Weges vor- } \\
& \text { überkam, ein jeder lobte und niemand tadelte sie' }
\end{aligned}
$$

Die GB wird als $\mathrm{E}=\mathrm{S}$ und die $\mathrm{PB}$ als $\mathrm{R}<\mathrm{S}$ gekennzeichnet. Die GB wird bei der Interpretation des Satzes getilgt.

\subsection{Atemporale Bedeutung}

Der Referenzzeitpunkt $\mathrm{R}$ wird bei der atemporalen Verwendung des ipf. Nicht-Prät. auf der Zeitachse E - R - S nicht lokalisiert. Die atemporale Bedeutung wird in generischen Kontexten (= praesens gnomicum) gebraucht:

Поспешишь, людей насмешишь 'Eile mit Weile'

Что посеешь, то и пожнешь

'Was der Mensch säet, das wird er ernten'

Ворон ворону глаз не выклюнет

'Eine Krähe hackt der anderen kein Auge aus'

Manchmal wird die atemporale Bedeutung mit einer modalen Bedeutung verknüpft:

Пеший конного не догонит (= не сможет догнать)

'Der zu Fuß Gehende holt den Reiter nicht ein'

На всех не угодишь (= нельзя угодить)

'Man kann es nicht jedem recht machen' 


\subsubsection{Aspektuelle Bedeutungen}

\subsubsection{Unmarkierte aspektuelle Bedeutung}

Die aspektuelle Bedeutung des pf. Nicht-Prät. auf der Zeitachse E - R - S wird als E $<$ R definiert. Das pf. Nicht-Prät. bezeichnet in der Regel eine konkrete Einzelhandlung (= konkretfaktische Bedeutung), die in der Zukunft abgeschlossen wird:

Мария сдаст свой последний зачет_КРеждеству

'Maria wird ihre letzte Prüfung vor Weihnachten ablegen'

\subsubsection{Markierte aspektuelle Bedeutungen}

Als markierte aspektuelle Bedeutungen werden die anschaulich-exemplifizierende Bedeutung bei der Bezeichnung multiplikativer Handlungen (s. Beispiele (3-4-9) und (3-4-10)) und die modalen ( $=$ die possibilitative und die nezessitative) Bedeutungen registriert.

Die possibilitative (= potenzielle) Bedeutung tritt dann auf, wenn Möglichkeit bzw. Unmöglichkeit der Realisierung einer Handlung ausgedrückt wird:

$$
\begin{aligned}
& \text { Петр всегда найдет (= сможет найти) выход } \\
& \text { 'Peter findet immer einen Ausweg '(= kann finden) } \\
& \text { Иван отличный слесарь. Он откроет (= сможет открыть) любой } \\
& \text { замок } \\
& \text { 'Ivan ist ein guter Schlosser. Er öffnet ein beliebiges Schloß' (= kann } \\
& \text { öffnen) } \\
& \text { С ним не nоговоришь (= нельзя поговорить) откровенно } \\
& \text { 'Mit ihm kann man nicht offen sprechen" } \\
& \text { Bсего не nерескажешь (=нельзя пересказать) } \\
& \text { 'Man kann nicht alles erzählen' }
\end{aligned}
$$

Das pf. Nicht-Prät. bringt manchmal die Notwendigkeit des Eintretens einer Handlung oder die Überzeugung des Sprechers, daß eine Handlung realisiert wird, zum Ausdruck:

$$
\begin{aligned}
& \text { Вода закипит (= должна закипеть), если температура } \\
& \text { достигнет } 100 \text { градусов Цельсия } \\
& \text { 'Das Wasser beginnt zu kochen, wenn die Temperatur } 100 \mathrm{Grad} \\
& \text { Celsius erreicht' } \\
& \text { Cтуденты npuдуm (= должны придти) завтра на экзамен } \\
& \text { 'Die Studenten werden morgen zur Prüfung kommen' }
\end{aligned}
$$




\subsection{Das imperfektive Präsens vom Typ paccказываю 'ich erzähle'}

\subsubsection{Temporale Bedeutungen}

\subsubsection{Unmarkierte temporale Bedeutung}

Das imperfektive Präsens (ipf. Präs.) vom Typ рассказываю 'ich erzähle' bezeichnet im unmarkierten Gebrauch eine gegenwärtige Handlung. Die temporal-grammatische Bedeutung (GB) und die temporal-pragmatische Bedeutung (PB) werden entsprechend als $\mathrm{E}=\mathrm{S}$ und $\mathrm{R}=$ $S$ dargestellt:

Петр сейчас пишет письмо

'Peter schreibt jetzt einen/den Brief

Der Prozeß des Schreibens (E) findet im Sprechzeitpunkt (S) statt, der mit dem Referenzzeitpunkt (R) ceйчac 'jetzt' identisch ist. Das ipf. Präs. weist die Bedeutung der negativen Spezifizierung des Merkmals [ $\alpha$ GETR] ([-GETR]) auf.

\subsubsection{Markierte temporale Bedeutungen}

Als markierte temporale Bedeutungen treten die präteritale Bedeutung (= praesens historicum), die futurale Bedeutung (= praesens propheticum) und die atemporale Bedeutung auf.

\subsection{Präteritale Bedeutung ( $\quad$ praesens historicum)}

Das ipf. Präs. der Narration, d. h. das historische Präsens, bezeichnet gegenwärtige Handlungen $(E=S)$, deren Referenzzeitpunkt $R$ in der Vergangenheit lokalisiert ist: $R<S$ :

В 1880 году работает Ф. М. Достоевский над романом "Братья Карамазовы"

'Im Jahre 1880 schreibt F. M. Dostojevski an dem Roman "Die Brüder Karamasov"

Вдруг двери отворились, Маша вбегает и с визгом кидается мне на шею (А. С. Пушкин)

'Plötzlich ging die Tür auf: Mascha stürzt herein und wirft sich mir mit einem Aufschrei an den Hals'

In der Verbindung mit der Partikel бывало bezeichnet das ipf. Präs. eine usuelle Handlung in ferner Vergangenheit:

Бэлла, бывало, нам поет песни или плящет лезгинку

(М. Ю. Лермонтов)

'Bella sang uns früher oft Lieder vor, oder sie tanzte die Lesginka' 


\subsection{Bedeutung des Futurs}

Das Präsens der beabsichtigten oder vorweggenommenen Handlung (= praesens propheticum) drückt die Bereitschaft des Sprechers zur Durchführung einer Handlung, die schon geplant ist, oder seine Überzeugung von der Realität einer Handlung, die vorauszusehen ist, aus:

Этим летом я еду за границу

'In diesem Sommer fahre ich ins Ausland'

Черезмесяц начинаютсл каникулы

'In einem Monat beginnen die Ferien'

Die adverbiellen Bestimmungen vom Тур этим летом 'in diesem Sommer', через месяц 'in einem Monat' weisen auf den entsprechenden Kontext hin.

Die GB wird als $E=S$ und die $P B$ als $R>S$ repräsentiert. Eine präsentisch aufgefaßte Handlung gilt dann als Ausdruck des Futurs.

\subsection{Atemporale Bedeutung}

Der atemporale Gebrauch des ipf. Präs. wird durch die Nicht-Spezifizierung des Referenzzeitpunkts $\mathrm{R}$ gekennzeichnet. Die Handlung wird dadurch auf der Zeitachse nicht lokalisiert. Das generische Präsens (oder praesens gnomicum) wird in wissenschaftlichen Texten bei der Feststellung von allgemein gültigen Gesetzmäßigkeiten und natürlichen Gegebenheiten (Beispiele (3-5-7) -(3-5-9)) und in literarischen Texten bei der Formulierung von Aphorismen und Sentenzen verwendet (Beispiele (3-5-10) und (3-5-11)).

Луна движется вокруг Земли по сложной незамкнутой орбите

Der Mond bewegt sich auf einer komplizierten, nicht geschlossenen

Umlaufbahn um die Erde'

Водород сам горит, а горения других веществ не поддержсивает

'Wasserstoff brennt selbst, aber fördert nicht die Verbrennung anderer

Stoffe'

Человек дышит легкими, а рыбы дышат жабрами

'Der Mensch atmet mit der Lunge, und die Fische atmen mit den Kiemen'

Кто много грозит, тот мало вредит

'Wer viel droht, schadet wenig'

Жизнь требует компромиссов

'Das Leben verlangt Kompromisse'

Das sogenannte praesens scenicum, das sich in Theaterstïcken und Drehbüchern als Bühnenanweisungen findet, bezeichnet auch zeitlose Handlungen: 
'Maria betritt das Zimmer, setzt sich auf einen Stuhl und beginnt ein Buch zu lesen'

Alle generischen Sätze lassen sich auf die Gegenwart, die Vergangenheit und auf die Zukunft gleichermaßen beziehen: Es sind die sogenannten ewigen Wahrheiten.

\subsubsection{Aspektuelle Bedeutungen}

\subsubsection{Unmarkierte aspektuelle Bedeutung}

Das ipf. Präs. bezeichnet eine konkrete Einzelhandlung, die in ihrem Verlauf dargestellt wird (= die konkret-prozessuale Bedeutung):

Петр играет в данный момент в футбол

'Peter spielt in diesem Augenblick Fußball'

Diese Bedeutung wird als primäre aspektuelle Bedeutung des ipf. Präs. interpretiert. Aspektuelle Bedeutung des ipf. Präs. auf der Zeitachse E- R- $\mathrm{S}$ wird als $\mathrm{E}=\mathrm{R}$ bestimmt.

\subsubsection{Markierte aspektuelle Bedeutungen}

Die Form des ipf. Präs. kann die folgenden mehrmaligen (oder multiplikativen) Handlungen bezeichnen: die begrenzt-iterative (Beispiel (3-5-14)), die unbegrenzt-iterative (Beispiel (3-515)), die usuelle (oder die habituelle) (Beispiel (3-5-16)), die permanente (oder die qualifizierende) (Beispiel (3-5-17)), die possibilitative (oder die potentielle) (Beispiel (3-5-18)).

Петр три раза перечитывает это стихотворение 'Peter liest diesesGedicht dreimal nacheinander Петр многе раз перечитывает это стихотворение 'Peter liest dieses Gedicht viele Male nacheinander Мария обычно по воскресеньям играет в теннис 'Maria spielt gewöhnlich sonntags Tennis Мария работает на фабрике 'Maria arbeitet in der Fabrik' Петр играет (= умеет играть) в футбол 'Peter spielt (= kann spielen) Fußball'

Die multiplikativen Handlungen werden als sekundäre oder markierte aspektuelle Bedeutungen des ipf. Präs. aufgefaßt. 

Ebene

Das System der aspektuell-temporalen Formen, das im Schema 1.1 angegeben ist, dient als Ausgangspunkt für die Ermittlung der Markiertheitsrelationen zwischen den eizelnen AspektTempus-Formen auf der paradigmatischen Ebene. Die Strukturierung der Aspekt-TempusFormen des modernen Russischen wird - wie im Schema $4.1 \mathrm{zu}$ sehen ist - nach den temporalen und aspektuellen Merkmalen [ $\alpha$ ANT(ERIOR)], [ $\alpha$ POST(ERIOR)], [ $\alpha$ GETR(ENNT HEIT)] und [ $\alpha$ TOT(ALITÄT)] vorgenommen. Das Zeichen $\alpha$ bezeichnet den nichtspezifizierten Wert des entsprechenden Merkmals; $\alpha$ kann die Bedeutung $+(=$ die positive Spezifizierung), die Bedeutung - (= die negative Spezifizierung) oder \pm (= die Neutralisierung des positiven und des negativen Wertes des Merkmals) annehmen. Das Zeichen u bedeutet 'unmarkiert', und das Zeichen $\mathrm{m}$ bedeutet 'markiert' in bezug auf die entsprechende aspektuell-temporale Form.

4.1 Paradigmatische Markiertheitsrelationen im System der aspektuell-temporalen Formen des modernen Russischen

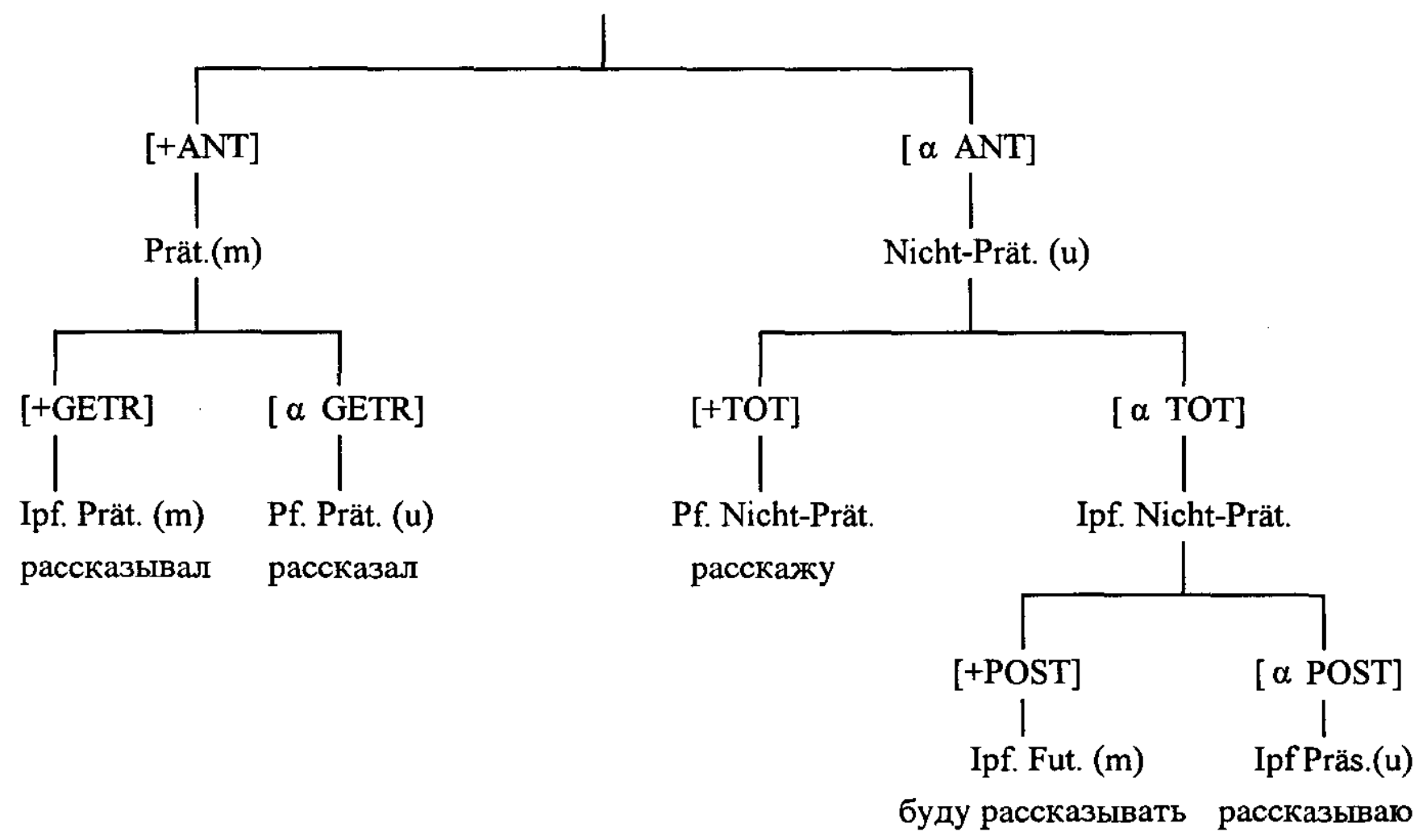

Das Paradigma des Präteritums als markiertes Glied der temporalen Opposition (m) mit positiver Spezifizierung des Merkmals der Vorzeitigkeit der Handlung [+ANT] und mit gesondertem Tempusmarker - $\mathbb{I}$ - ist den Paradigmen des Nicht-Präteritums mit nichtspezifiziertem Wert des Merkmals der Vorzeitigkeit [ $\alpha$ ANT] gegenübergestellt. Innerhalb des präteritalen Bereiches besteht die Opposition zwischen imperfektivem und perfektivem Präteritum. Das imperfektive 
Präteritum vom Typ рассказывал 'er erzählte' mit dem Merkmal [+GETR] symbolisiert die vollständige Trennung zwischen der vergangenen Handlung und dem Sprechzeitpunkt (vgl. Abschnitt 3.1.1.1), während das perfektive Präteritum vom Тур рассказал 'er hat erzählt' den Bruch zwischen der Handlung und dem Sprechzeitpunkt offen läßt ([ $\alpha$ GETR]) (vgl. Abschnitt 3.2.1.1). Das perfektive Präteritum kann deshalb sowohl die Bedeutung des alten Aorists (= die Bezeichnung der vollständigen Trennung des Ereignisses vom Sprechzeitpunkt $)(=[+G E T R])$ als auch die Bedeutung des Perfekts (= die Bezeichnung der vergangenen Handlung, die zum Sprechzeitpunkt aktuell ist) (= [-GETR]) ausdrücken. Das imperfektive Präteritum mit dem Merkmal der Getrenntheit (= [+GETR]) tritt deshalb als markiertes Glied der aspektuell-temporalen Korrelation imperfektives Präteritum - perfektives Präteritum auf.

Im nichtpräteritalen Bereich wird die Form des perfektiven Nicht-Präteritums vom Typ pacскажу mit dem Merkmal der Totalität der Handlung ([+TOT]) den Formen des imperfektiven Nicht-Präteritums gegenübergestellt. Die Gliederung ist wie auch im linken Teil des Schemas nach Aspektformen vorgenommen. Als Grundlage der Klassifizierung dient im linken Teil des Schemas das Merkmal [ $\alpha$ GETR], während im rechten Teil des Schemas das Merkmal [ $\alpha$ TOT] fungiert. Die Verteilung der Markiertheitswerte bei den aspektuellen Formen des Präteritums und des NichtPräteritums ist dementsprechend unterschiedlich. Die darauf folgende Opposition [+POST] / [ $\alpha$ POST] trennt das Futur vom Präsens im Rahmen des imperfektiven Nicht-Präteritums. Die Form des imperfektiven Futurs vom Тур буду рассказывать 'ich werde erzählen' mit dem Merkmal der Nachzeitigkeit der Handlung im Vergleich zum Sprechzeitpunkt ([+POST]) bildet das markierte Glied dieser Opposition, während die Form des Präsens vom Typ рассказываю 'ich erzähle' mit nichtspezifiziertem Wert des Merkmals der Nachzeitigkeit ([ $\alpha$ POST]) unmarkiert bleibt.

Alle drei aspektuell-temporalen Formen des Nicht-Präteritums sind einander gegenübergestellt. Im Schema 4.1 wird die Korrelation des imperfektiven Futurs vom Тур буду рассказывать 'ich werde erzählen' mit dem Merkmal [+POST] und des imperfektiven Präsens vom Typ рассказываю 'ich erzähle' mit dem Merkmal[ $\alpha$ POST]) repräsentiert. Diese beiden Formen werden ihrerseits der Form des perfektiven Nicht-Präteritums vom Tyр расскажу gegenïbergestellt. Diese drei Oppositionen werden explizit im Schema 4.2 angegeben. 


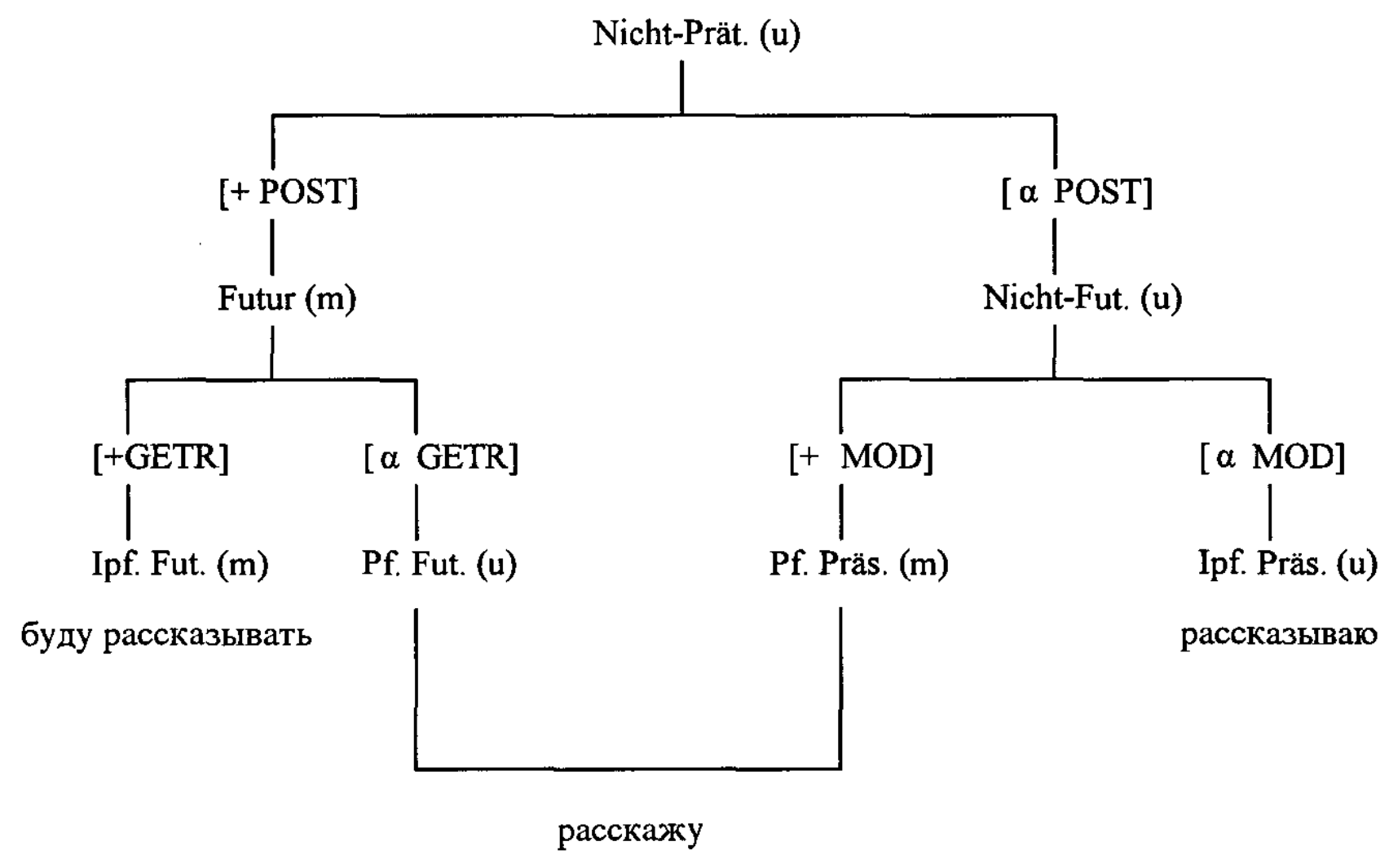

Die ersten zwei Oppositionen werden im Rahmen des Futurs bzw. des Nicht-Futurs (d. h. Präsens) als aspektuelle Oppositionen aufgefaßt. Das perfektive Nicht-Präteritum vom Typ расскажу in der Bedeutung des Futurs (= 'ich werde erzählen') wird dem imperfektiven Futur vom Тур буду рассказывать gegenübergestellt. In dieser ersten Korrelation tritt das imperfektive Futur mit dem Merkmal der Getrenntheit der Handlung ([+GETR]) und mit dem separaten Futurmarker (das Hilfsverb буду + Infinitiv) als markiertes Glied auf, vgl. Abschnitt 3.3.1.1. Das perfektive Nicht-Präteritum vom Typ расскажу wird als unmarkiertes Glied interpretiert. Die gleiche Form des perfektiven NichtPräteritums vom Typ расскажу wird als Präsens (= 'ich erzähle') dem imperfektiven Präsens vom Typ рассказываю gegenübergestellt. In dieser zweiten Korrelation weist das perfektive NichtPräteritum vom Typ pасккажу als markiertes Glied die Bedeutung der Modalität der Handlung ([+MOD(ALITÄT)]) auf, vgl. Abschnitt 3.4.1.2.1. Die temporalen Bedeutungen des unmarkierten perfektiven Futurs und des markierten perfektiven Präsens werden formal im Rahmen des perfektiven Nicht-Präteritums nicht auseinandergehalten. Sprachhistorisch gesehen war die Bedeutung des Präsens primär. Mit der Ausprägung der Aspekte verschiebt sich die Bedeutung dieser Form immer mehr in Richtung des Futurs. Die Bedeutung der Zukunft ist im modernen Russischen dominant. Die Bedeutung des Präsens ist dagegen peripher.

Die dritte Korrelation des imperfektiven Futurs vom Тур буду рассказывать 'ich werde erzählen' und des imperfektiven Präsens vom Тур рассказываю 'ich erzähle' wird im Schema (4.2) als Gegenüberstellung dieser Formen nach dem obersten temporalen Merkmal [+POST]/ $[\alpha$ POST] $)$ aufgefaßt. Diese letzteren Formen bewahren ihre temporale Bedeutung auch in den 
Oppositionen mit dem perfektiven Präteritum vom Typ pacскажу. Die doppelte temporale Etikettierung der Form vom Typ paccкажу veranlaßt uns, diese Form paradigmatisch als perfektives NichtPräteritum mit Neutralisierung der temporalen Bedeutungen des Präsens und des Futurs im Rahmen des markierten perfektiven Aspekts zu interpretieren, vgl. die Neutralisienung der temporalen Differenzierung innerhalb der markierten Modi (des Konjunktivs und des Imperativs) oder die NichtUnterscheidung des Genus im Rahmen des markierten Plurals bei Substantiven und Adjektiven. Dadurch wird die Optimierung des Aspekt-Tempus-Systems erreicht: Anstatt der 6 AspektTempus-Formen treten nur 5 auf.

\section{$5 \quad$ Fazit}

In diesem Beitrag werden temporale, aspektuelle und modale Bedeutungen der AspektTempus-Formen des modernen Russischen untersucht. Die Analyse wird unter dem Blickwinkel des Konzepts der Markiertheit durchgeführt (vgl. Wurzel 1998: 62-65). Solches Herangehen gestattet es, die in der Russistik existierende Meinungen über den Status der Form vom Тур расскажу zu revidieren und auf dieser Basis die strukturellen Beziehungen zwischen den einzelnen aspektuell-temporalen Formen neu zu definieren, die Organisation dieser Formen als optimales System zu erklären und die Mechanismen des Funktionierens der aspektuell-temporalen Formen aufzudecken

Die Markiertheitsrelationen werden sowohl auf der paradigmatischen Ebene, d. h. auf der Ebene der Relationen einzelner Aspekt-Tempus-Formen zueinander, als auch auf der syntagmatischen Ebene, d. h. auf der Ebene des Gebrauchs jeder einzelnen aspektuell-temporalen Form in verschiedenen Kontexten behandelt.

Auf der paradigmatischen Ebene wird die systemorganisierende Funktion der Markiertheitsrelationen demonstriert. Der ipf. A. als unmarkiertes Glied der aspektuellen Opposition mit der Nicht-Signalisierung des Merkmals der Totalität der Handlung ([ $\alpha$ TOT $]$ ) hat alle drei Tempusparadigmen (Präteritum, Futur und Präsens). Der pf. A. als markiertes Glied der Aspektopposition mit dem Merkmal der Signalisierung der Totalität der Handlung ([+TOT]) weist nur zwei Typen der Tempusparadigmen, nämlich das Präteritum und das Nicht-Präteritum auf: Das Präsens und das Futur werden im Rahmen des markierten pf. A. neutralisiert. Durch die Vereinigung der Bedeutungen des Futurs und des Präsens in einer Form wird die Ökonomie im System der aspektuell-temporalen Formen erreicht: Anstatt zweier Formen (des Futurs und des Präsens) tritt im perfektiven Aspekt nur eine einzige Form, nämlich die Form des pf. NichtPrät. auf.

Die Unterscheidung unmarkierter und markierter Dimensionen auf der syntagmatischen Ebene erlaubt die Trennung des Zentrums des Sprachsystems von der Peripherie: Die unmarkierten Bedeutungen sind primär oder zentral, während die markierten Bedeutungen sekundär, peripher oder marginal sind. 


\section{Literatur}

Bybee, J. L. \& Dahl, Ö. (1989): The creation of tense and aspect systems in the languages of the world. Studies in Language 13-1, 51-103.

Guiraud-Weber, M. (1988): L'aspect du verbe russe (Essais de présentation). Aix-enProvence.

Isačenko, A. V. (1976 [1960]): La structure sémantique des temps en russe, in: Isačenko, A. V. (1976): Opera selecta. München, 215-229.

Isačenko, A. V. (1962): Die russische Sprache der Gegenwart. Teil I: Formenlehre. Halle (Saale).

Kiparsky, P. (1998): Aspect and Event Structure in Vedic. In: The Yearbook of South Asia Languages and Linguistics 1998. New Delhi, 29-61.

Reichenbach, H. (1966): Elements of Symbolic Logic. New York/London.

RSG 1988: Russische Sprache der Gegenwart. Bd. 2: Morphologie. Leipzig 1988.

Wurzel, W. U. (1998): On markedness. Theoretical Linguistics, Vol. 24, No 1, 53-71.

Виноградов, В. В. (1972): Русский язык (грамматическое учение о слове). Издание второе. Москва.

ГСРЛЯ (1970): Грамматика современного русского литературного языка. Москва.

Домбровский, Й. (1971): Историческая грамматика русского языка II: Морббология и синтаксис. Будапешт.

Рассудова, О. ПІ. (1982): Употребление видов глагола в современном русском лзыке. Москва.

РГ 1979: Русская Грамматика 1. Praha 1979.

РГ 1980: Русская грамматика, т. І. Москва 1980. 